\title{
Post-translational events in the intracellular transit of apolipoprotein-B: modulation by dietary lipids
}

\author{
Joan A. Higgins*, John Kendrick, Jane Wilkinson and Ian Cartwright \\ Department of Molecular Biology and Biotechnology, University of Sheffield, Sheffield S10 2TN, UK
}

Triacylglycerol and cholesteryl esters are transported in the blood as components of the plasma lipoproteins. Endogenous triacylglycerol synthesized by the liver is secreted into the plasma in the form of VLDL, which consists of a droplet of non-polar lipid (predominantly triacylglycerol with a variable amount of cholesteryl ester), stabilized by an outer shell of phospholipid, cholesterol and protein. Apolipoprotein-B (apo-B) is the major protein of VLDL. Although apo-B is an essential component of VLDL, secretion is driven by provision of substrates to the liver for triacylglycerol synthesis. These include glucose and nonesterified fatty acids. The composition and size of VLDL is influenced by the substrate provided (Sniderman \& Cianflone, 1993). Dietary experiments and experiments using isolated hepatocytes have shown that provision of carbohydrates increases synthesis of triacylglycerol-enriched, larger, lighter VLDL (VLDL1), while provision of fatty acids results in production of smaller, denser VLDL (VLDL2). Since VLDL1 are cleared rapidly from the circulation, while VLDL2 are cleared more slowly and are converted to the atherogenic LDL, the nature of VLDL secreted by the liver is an important determinant of cardiovascular risk (Packard et al. 1984; Shepherd \& Packard, 1987). An understanding of the mechanisms involved in regulation of the assembly of VLDL and the factors determining their composition is, therefore, extremely important.

\section{Apolipoprotein-B secretion is regulated post-translationally}

Under conditions in which apo-B secretion by hepatocytes varies up to sevenfold there is no significant change in the mRNA for apo-B (Kosykh et al. 1988; Pullinger et al. 1989). A number of studies on isolated hepatocytes and hepatoma cells have shown that apo-B is synthesized in excess of that secreted, and that the excess protein is degraded intracellularly (Boren et al. 1990, 1993; Davis et al. 1990; Sato et al. 1990; Dixon et al. 1991; White et al. 1992; Cartwright et al. 1993; Dixon \& Ginsberg, 1993; Cartwright \& Higgins, 1996). This has led to the idea that apo-B is synthesized constitutively and in excess; the protein not assembled into VLDL being degraded. Thus, availability of lipid drives VLDL secretion by diverting apo-B from degradation to secretion. Post-translational intracellular transit of apo-B, therefore, plays a major role in the regulation of VLDL secretion.

\section{Experimental models for investigation of assembly and secretion of VLDL}

Most studies of apo-B secretion have been carried out using hepatoma cell lines. However, although these investigations have yielded valuable results, hepatoma cells do differ significantly from normal hepatocytes. The most popular model, Hep-G2 cells, secrete lipid-poor lipoprotein particles of greater density than that of LDL (Gibbons, 1994). McArdle cells, a rat hepatoma cell line, secrete both apoB48 and apo-B100, while human liver secretes only apoB100 (Boren et al. 1994). Because we wish to extrapolate our findings to human subjects and investigate factors such as diet and hormonal control of VLDL secretion, we have opted to use animal models for our studies. Both the rabbit and the hamster liver secrete VLDL containing apo-B100 similar to those secreted by the human liver, and the subsequent metabolism of VLDL is similar to that in human subjects. We have, therefore, used rabbit and hamster for the studies described in the present review. Our basic experimental protocol is to prepare isolated hepatocytes, to incubate these under defined conditions with radiolabelled precursors of apo-B $\left({ }^{35} \mathrm{~S}\right]$ methionine $)$ and lipids $\left({ }^{3} \mathrm{H}\right.$ or $\left.{ }^{14} \mathrm{C}\right]$ glycerol, oleate or mevalonate), and to follow the intracellular transit of apo-B and lipid through the secretory compartment by preparing subcellular fractions (rough endoplasmic reticulum (RER), smooth endoplasmic reticulum (SER), cis-Golgi and trans-Golgi), for analysis (Cartwright \& Higgins, 1992, 1995, 1996; Wilkinson et al. 1992, 1993; Cartwright et al. 1993, 1997).

\section{A two-step model for the assembly of VLDL}

A model for VLDL assembly which takes into account our work and that of other laboratories is illustrated in Fig. 1. Newly-synthesized apo-B is either transferred across the

\footnotetext{
Abbreviations: ALLN, N-acetyl-leucyl-leucyl-norleucinal; apo-B, apolipoprotein-B; MTP, microsomal triglyceride transfer protein; RER, rough endoplasmic reticulum; SER, smooth endoplasmic reticulum.

*Corresponding author: Dr Joan Higgins, fax + $44(0) 1142728697$
} 


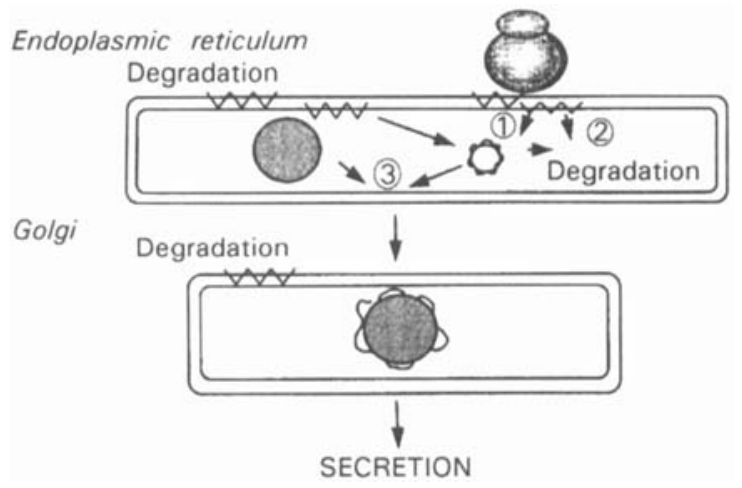

Fig. 1. A model for the assembly of VLDL in hepatocytes. W,

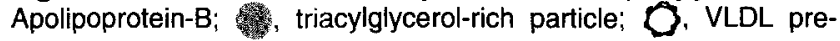
cursor.

endoplasmic reticulum membrane or remains membrane bound. Using a panel of monoclonal antibodies, we have shown that in the RER and SER the membrane-bound form is in two pools, one available to probes from the cytosolic side, and the second only available in permeabilized membranes (Wilkinson et al. 1993). In the Golgi, the whole membrane-bound pool is at the cytosolic side of the membrane. The membrane-bound apo- $\mathrm{B}$ is degraded mainly in the RER; however, if degradation is inhibited, the protein accumulates in the trans-Golgi membrane (Cartwright et al. 1993; Cartwright \& Higgins, 1996). The ubiquitin-proteosome pathway has been implicated in this degradation step (Yeung et al. 1996). The lumen pool of apo-B is associated with only a small amount of triacylglycerol, cholesterol and cholesteryl ester (Cartwright et al. 1997). A large proportion of this pool of apo-B is also degraded in the RER (Cartwright et al. 1993). The bulk of the lipids, triacylglycerol, cholesterol and cholesteryl esters of VLDL are transferred into the lumen of the SER and become associated with apo-B at some point between the SER and the cis-Golgi (Cartwright \& Higgins, 1995; Cartwright et al. 1997).

Triacylglycerol-rich particles, which lack apo-B, have been observed in the SER lumen and have also been isolated by subcellular fractionation, suggesting that the VLDL lipids may be added in a single step to the apo-Bcontaining precursor particles (Higgins \& Hutson, 1984). In this model there are three potential regulatory steps: (1) transfer of apo-B across the RER membrane into the lumen. This pool may be transiently associated with the luminal surface of the endoplasmic reticulum membrane; (2) assembly of apo-B into a VLDL precursor. As considerable degradation of the newly-synthesized apo-B occurs in the lumen of the RER, these precursor particles may be heterogeneous and only a proportion move on to be secreted; (3) the VLDL precursors move to the SER lumen and fuse with triacylglycerol-rich particles to form a VLDL particle for secretion.

\section{Translocation of apolipoprotein-B across the membrane of the rough endoplasmic reticulum}

Several recent studies on Hep-G2 cells have significantly contributed to the understanding of molecular events in

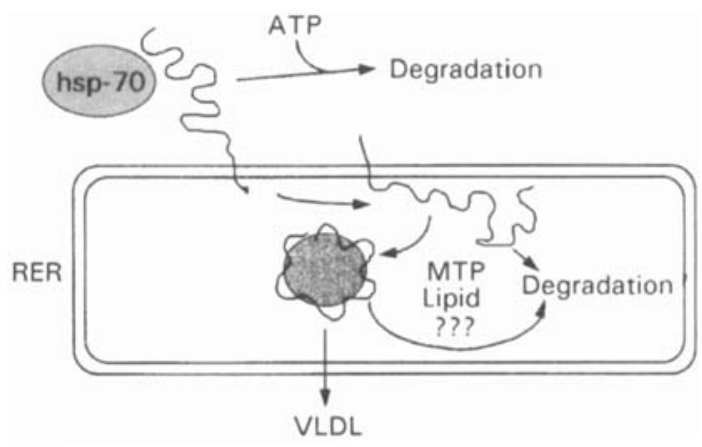

Fig. 2. Translocation of apolipoprotein-B across the endoplasmic reticulum. RER, rough endoplasmic reticulum; hsp 70, heat shock protein 70; MTP, microsomal triglyceride transfer protein.

apo-B translocation. These observations are incorporated into the model shown in Fig. 2. Apo-B is a large (molecular mass $500000 \mathrm{kD}$ ) hydrophobic protein. It apparently undergoes translation arrest when only a small part of the protein is at the luminal side of the RER membrane (Du et al. 1994). Synthesis of the protein is completed, leaving a large part of the polypeptide at the luminal surface. At this stage the protein is not integrated into the membrane and is presumably still associated with the translocon complex. Apo-B is stabilized by transient association with the cytosolic heat shock protein 70 (Zhou et al. 1995). In the presence of ATP, heat shock protein 70 dissociates and apo-B is degraded. In the presence of oleate, apo-B is translocated into the RER lumen. This may involve a transient membrane-bound pool in which the bulk of apo-B is at the luminal side of the membrane (Wilkinson et al. 1993). Apo-B is transiently associated with microsomal triglyceride transfer protein (MTP; Wu et al. 1996), which in vitro transfers triacylglycerol and cholesteryl ester between membranes (Gordon et al. 1995; Jamil et al. 1996). Inhibition of MTP blocks the early steps in VLDL assembly and MTP, therefore, may transfer stabilizing lipid to the apo-B molecule on translocation into the RER lumen (Gordon et al. 1996). Apo-B that is not properly folded or stabilized by lipids is degraded (Cartwright et al. 1993). The first steps in the transfer of apo-B into the RER lumen are thus complex and may involve MTP, specific lipids, and other, as yet unidentified, factors.

\section{Effect of dietary lipids on VLDL secretion}

Provision of fatty acids to the liver results in the production of small dense VLDL. A diet enriched in fish oils (containing the $n-3$ fatty acids, docosahexaenoic acid and eicosapentaenoic acid) reduces plasma triacylglycerol levels by reducing secretion of VLDL by hepatocytes (Lang \& Davis, 1990; Wang et al. 1993). To investigate the way in which dietary fat affects VLDL assembly, rabbits or hamsters were fed on either normal chow $(25 \mathrm{~g}$ fat $/ \mathrm{kg})$ or chow supplemented with $100 \mathrm{~g}$ sunflower oil (high $n-6$ fatty acids) $/ \mathrm{kg}$ or with $100 \mathrm{~g}$ fish oil (high $n-3$ fatty acids) $/ \mathrm{kg}$. In order to ensure that the same amount of fat was consumed, and to overcome the reluctance of rabbits to eat food to which oil had been added, the oils were administered orally 
on a daily basis. The fatty acid compositions of these diets were extreme compared with the human diet. However, this regimen was adopted to determine whether an effect could be detected. The diets would then be altered to one closer to that of the human diet for further study. In both rabbits and hamsters, dietary fish oils reduced the levels of plasma VLDL compared with chow-fed animals. Dietary sunflower oil had no significant effect on VLDL or triacylglycerol levels.

\section{Intracellular pools of apolipoprotein-B are altered by different diets}

The apo-B content (per $\mathrm{mg}$ protein in the fraction) was highest in the trans-Golgi fraction, consistent with the fact that VLDL particles accumulate in these vesicles (Fig. 3). The major effect of dietary fish oils was to reduce the apo$\mathrm{B}$ content of the trans-Golgi fraction by more than $50 \%$. Both sunflower-oil and fish-oil diets resulted in a large increase in the apo-B content of the RER (Fig. 3). When the subcellular fractions were separated into membranes and lumen contents, further effects of diet on the distribution of apo-B pools were observed. (Fig. 3). In endoplasmic reticulum, RER and SER fractions from chow-fed animals approximately half the apo-B was membrane bound. In fractions from livers of both fish-oil-fed and sunflower-oilfed animals, the membrane-bound pool was reduced and $90-95 \%$ of the apo-B was found in the lumen. In endoplasmic reticulum fractions from chow-fed animals, the membrane-bound apo-B was in two pools, one of which is available to probing with monoclonal antibodies

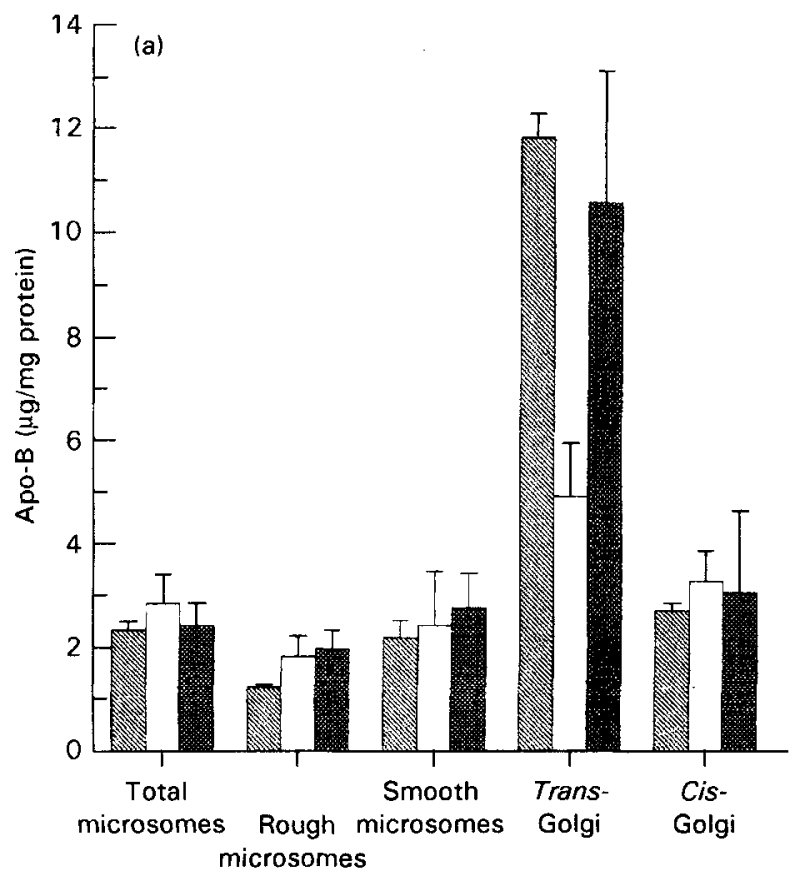

(cytosolic pool) and the second of which is only available to probes when the vesicles are opened (luminal pool). In endoplasmic reticulum fractions from oil-fed animals, all the apo-B in the membrane was available to probes and, therefore, is cytosolic. In trans-Golgi from the livers of chow-fed animals, $75 \%$ of the apo-B was luminal. In transGolgi from the livers of sunflower-oil-fed animals the lumen pool of apo-B was increased by approximately $25 \%$, while in the livers of fish-oil-fed animals the trans-Golgi lumen pool of apo-B was reduced by more than $50 \%$ (Fig. 3).

These observations suggest that a fat-enriched diet facilitates transfer of apo-B across the RER membrane into the secretory pathway. Dietary fish oils inhibit the further transfer of lumen apo-B from the endoplasmic reticulum to the trans-Golgi, while dietary sunflower oils increase this transfer. To determine whether lumen apo-B is associated with lipids under the different dietary conditions, the lumen fractions were concentrated, adjusted to a density of 1.21 with $\mathrm{KBr}$ and layered beneath a discontinuous $\mathrm{KBr}$ gradient made up of steps of density 1.005, 1.02 and $1.09 \mathrm{~g} / 1$. In all lumen fractions from the livers of fish-oilfed animals, apo-B remained in the density $1.21 \mathrm{~g} / 1$ layer. Dietary fish oil, therefore, inhibits association of lumen apo-B with lipid, consistent with the observation that fish oils inhibit VLDL secretion by the liver. In trans-Golgi lumen fractions from chow-fed and sunflower-oil-fed animals, $>80 \%$ of the apo-B floated towards the top of the gradient. In fractions from sunflower-oil-fed animals, the apo-B-containing particles were of higher mean density than in those from the chow-fed animals.

Fig. 3. (a) Intracellular pools of apolipoprotein-B (apo-B; $\mu \mathrm{g} / \mathrm{mg}$ protein) in subcellular fractions prepared from livers of rabbits fed on chow ( $\mathbb{N}$ ), or fish-oil $(\square)$ - or sunflower-oil ( $\square$ )-enriched diets (for details, see Wilkinson et al. 1992, 1993). The apo-B content was determined by ELISA (Wilkinson et al. 1993). (b) Membrane and lumen apo-B contents respectively in subcellular fractions prepared from livers of rabbits fed on chow

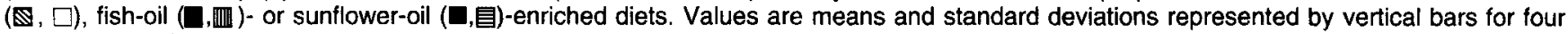
separate experiments. 


\section{Effect of diets on the kinetics of apolipoprotein-B degradation and secretion}

To determine whether dietary lipids modulate the intracellular transit of apo-B, hepatocytes were isolated from livers of hamsters fed on chow, or chow supplemented with sunflower oil or fish oil, and were incubated with $\left[{ }^{35} \mathrm{~S}\right]$ methionine to label apo-B pools. The cells were isolated by centrifugation and re-incubated with an excess of unlabelled methionine for a range of time periods. In this protocol, apo-B synthesized in the first incubation step was radiolabelled by incorporation of $\left[{ }^{35} \mathrm{~S}\right]$ methionine (pulse step). The fate of the radiolabelled apo-B was then followed by incubation with unlabelled methionine (chase step). Cells were re-isolated and in some cases subcellular fractions prepared. Total cell protein, media protein or subcellular proteins were separated by SDS-PAGE and the radioactivity in apo-B was determined. Using this protocol, the rate of secretion and/or intracellular degradation of apo-B was followed. In parallel experiments, the incorporation of radiolabelled methionine into total TCA-precipitated proteins was determined. This demonstrated that the cells were viable and were able to synthesize proteins, and that there was no further synthesis of total radiolabelled protein during the chase step. The rate of secretion of total radiolabelled protein was similiar in hepatocytes from chow-fed, sunflower-oil-fed and fish-oil-fed animals, and there was no degradation of the radiolabelled proteins during the chase step.

Approximately $55 \%$ of the newly-synthesized apo-B in chow-fed cells were degraded during the $120 \mathrm{~min}$ chase step and $9 \%$ of the protein was secreted (Fig. 4). In hepatocytes from sunflower-oil-fed hepatocytes, secretion was increased to $20 \%$ and, overall, $45 \%$ of the newlysynthesized apo-B was degraded (Fig. 4). In hepatocytes from fish-oil-fed animals, secretion was less than $5 \%$ and more than $90 \%$ of the radiolabelled intracellular apo-B was degraded (Fig. 4). Dietary sunflower oil thus targets apo-B into the secretory pathway, while dietary fish oils inhibit secretion and target apo-B into the degradative pathway.

\section{Effect of protease inhibitors on the intracellular transit of apolipoprotein- $B$}

$o$-Phenanthroline, a metalloprotease inhibitor, inhibits intracellular degradation of apo-B and results in accumulation of apo-B in the RER membrane (Cartwright \& Higgins, 1996). Secretion of apo-B is stimulated, suggesting that $\sigma$-phenanthroline protects a secretion-competent pool of apo-B from degradation. N-Acetyl-leucyl-leucylnorleucinal ( $A L L N)$, a cysteine protease inhibitor, inhibits degradation of apo-B and results in accumulation of apo-B in the trans-Golgi membrane. ALLN does not stimulate secretion of apo-B, suggesting that this inhibitor protects apo-B already committed to the degradative pathway. The use of these inhibitors thus provides a tool for probing the site of degradation in hepatocytes from fish-oil-fed animals.

$o$-Phenanthroline inhibited degradation and stimulated secretion of newly-synthesized apo-B in chow-fed animals (Fig. 4). ALLN also inhibited degradation but had little effect on secretion. In hepatocytes from fish-oil-fed animals, ALLN had little effect on either degradation or secretion of newly-synthesized apo-B. However, $o$-phenanthroline both stimulated secretion and inhibited degradation of newly-synthesized apo-B and reversed the effect of feeding fish oils. The effects of ALLN and $o$-phenanthroline on the fate of newly-synthesized apo-B was less marked in hepatocytes from sunflower-oil-fed animals. In this case, hepatocytes are primed for secretion of apo-B in the absence of inhibitors. Both $o$-phenanthroline and ALLN reduced degradation, and $o$-phenanthroline stimulated secretion by about $10 \%$.

In fish-oil-fed hepatocytes, apo-B is directed towards the degradation pathway. However, this is reversed by $o$-phenanthroline and apo-B secretion is stimulated. To determine whether the apo-B secreted under these conditions was in a particle of the density of VLDL, the incubation medium after $120 \mathrm{~min}$ was subjected to sequential centrifugation by adjusting the density to $1.019,1.063$ and $1.200 \mathrm{~g} / 1$ with $\mathrm{KBr}$. More than $90 \%$ of the apo-B was in the density of VLDL (density $<1.019 \mathrm{~g} / 1$ ). This was also found in similar experiments using hepatocytes from sunflower-oil-fed and chow-fed animals, without inhibitors.

Effect of provision of oleate in vitro on the fate of newlysynthesized apolipoprotein- $B$

To test whether provision of oleate during synthesis of apo$B$ affects its subsequent fate, a series of experiments were carried out in which oleate was present and absent in the pulse and chase steps (Fig. 5). When oleate was present in the pulse step, a greater proportion of apo-B was secreted than when oleate was omitted from the pulse step. When oleate was added in the chase step, this had no effect on the secretion of newly-synthesized apo-B but protected intracellular apo-B from degradation. Availability of oleate during synthesis of apo-B thus appears to direct a greater proportion of newly-synthesized apo-B into the secretory pathway. Addition of oleate in the chase step protects apo$B$ against degradation but does not increase its secretion, suggesting that a pool of apo-B not destined for secretion is protected.

\section{Conclusions}

The intracellular fate and transit of apo-B is complex. Newly-synthesized apo-B is either secreted or degraded. Apo-B not transferred into a secretory pool is degraded by an ALLN-sensitive process. Apo-B that has been directed into the secretory pool is degraded by an $o$-phenanthrolinesensitive process. The flux of apo-B through these pathways is affected by lipids. A lipid-enriched diet results in a greater proportion of apo-B in the lumen of the RER, the secretory pool. Provision of oleate in vitro during apo-B synthesis also directs apo-B into a secretory pool. Lipids therefore facilitate the movement of apo-B across the RER membrane and through the secretory pathway. However, a fish-oil-enriched diet inhibits movement of apo-B from the RER to the trans-Golgi and also inhibits transfer of lipid to the apo-B-containing particles, indicating that translocation 
Cellular degradation

(a) Chow-fed

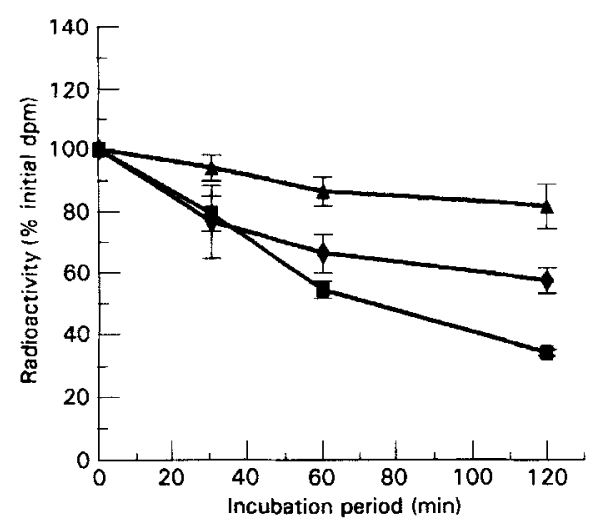

(b) Fish-oil-fed

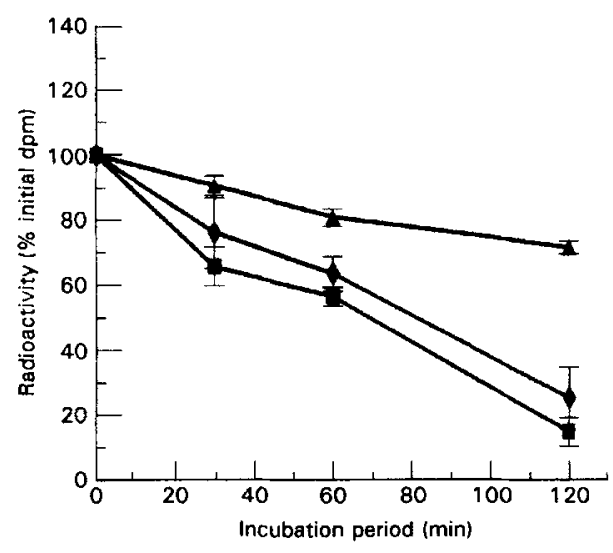

(c) Sunflower-oil-fed

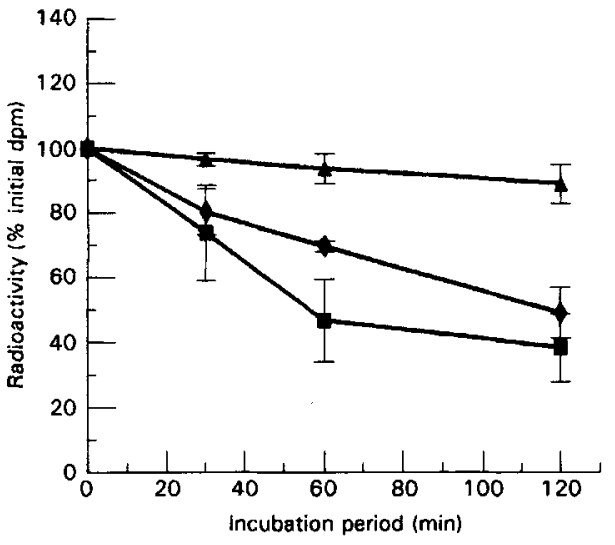

Secreted
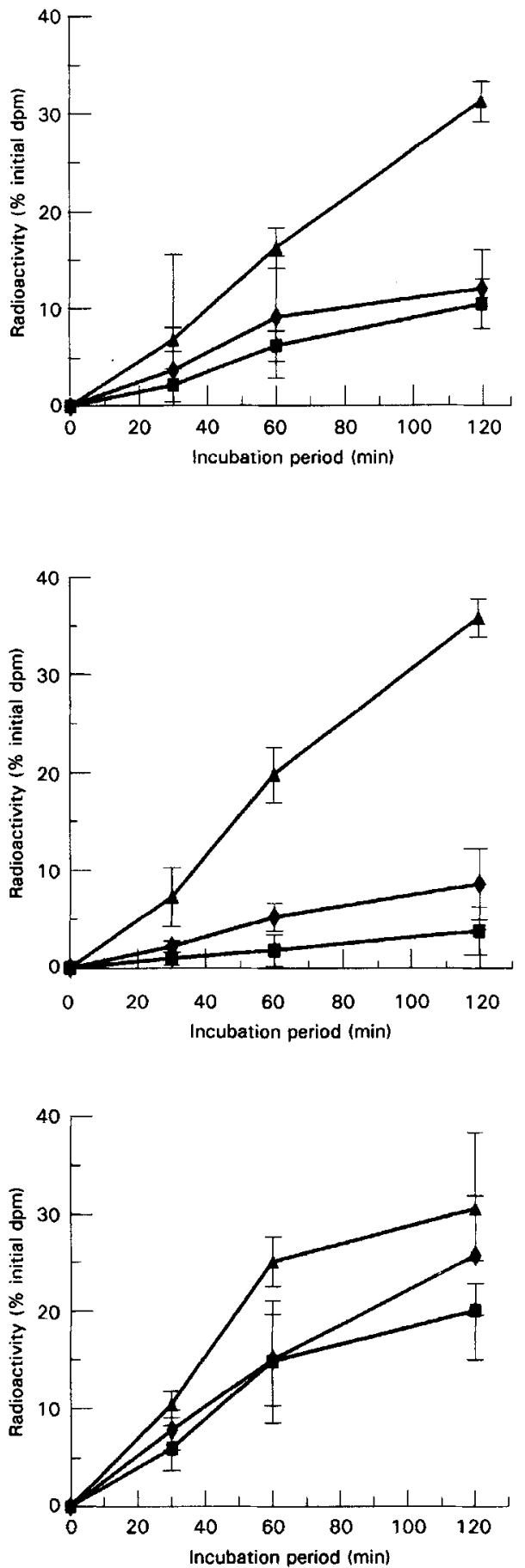

Fig. 4. Fate of newly-synthesized apolipoprotein-B (apo-B) in hepatocytes prepared from hamsters fed on different diets. Procedures were as described previously for rabbit hepatocytes (Cartwright et al. 1993; Cartwright \& Higgins, 1995, 1996). Hepatocytes were isolated from hamsters fed on (a) chow, or (b) fish-oil- or (c) sunflower-oil-enriched diets. The cell proteins were labelled by incubation with [ $\left.{ }^{35} \mathrm{~S}\right] \mathrm{methionine}$ for 30 min (pulse step). The hepatocytes were isolated by centrifugation and re-incubated with an excess of unlabelled methionine (chase step). At timed intervals the cells were re-isolated by centrifugation. The proteins of the cells and media were separated by SDS-PAGE and the radioactivity (disintegrations/min; dpm) in apo-B was determined either by cutting the apo-B band from the gel and digesting and measuring the radioactivity, or using an Instant Imager (Packard, Pangbourne, Berks.). Apo-B was identified by including an LDL-protein marker on each gel. To compare different preparations, the results are expressed as the percentage change in cellular and secreted apo- $\mathrm{B}$ assuming that radioactivity (dpm) in apo-B of the cells is $100 \%$ at the beginning of the chase period. Radioactivity (dpm) in cellular apo-B at the beginning of the chase was $150000-$ $600000 \mathrm{dpm}$. The variation is due to decay of the $\left[{ }^{35}\right.$ S]methionine. (a), No addition; ( $\bullet$ ), $\mathrm{N}$-acetyl-leucyl-leucyl-norleucinal; (A), o phenanthroline. Values are means and standard deviations represented by vertical bars for three separate experiments performed in duplicate. 
Secreted

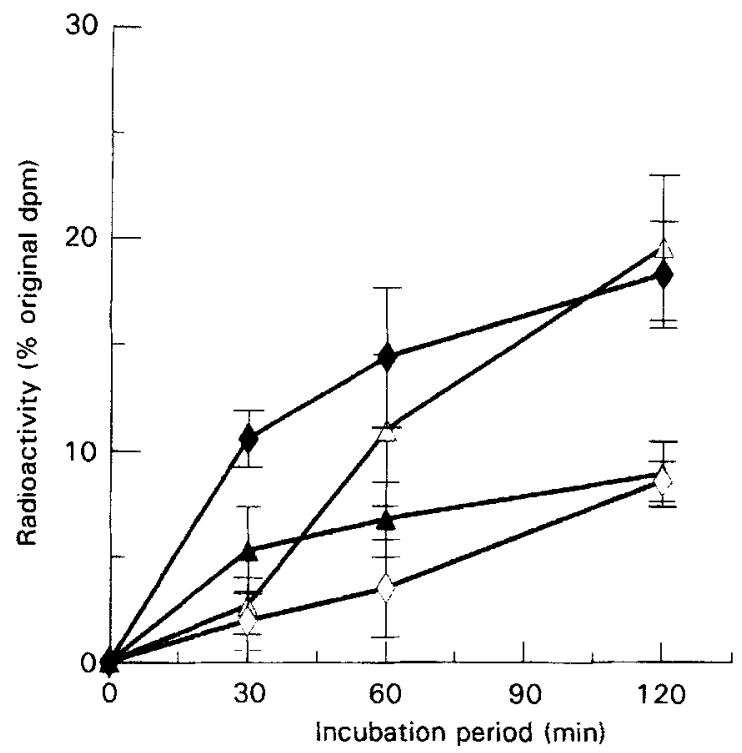

Cellular degradation

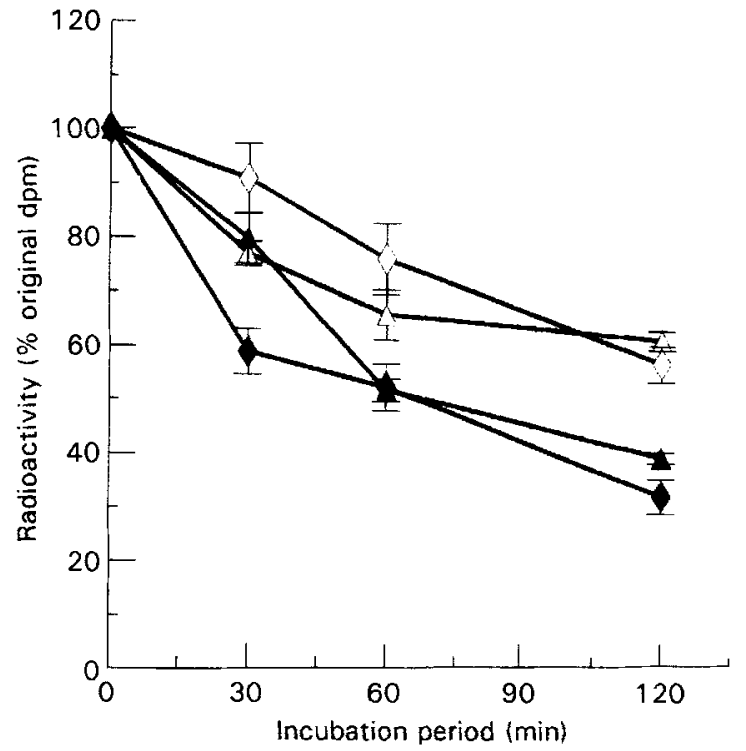

Fig. 5. Effect of oleate on the fate of newly-synthesized apolipoprotein-B in hamster hepatocytes. Procedures were as described previously for rabbit hepatocytes (Cartwright et al. 1993; Cartwright \& Higgins, 1995, 1996) except that oleate (final concentration 1 mM) was added in the puise step (apo-B labelled by incubation with [ ${ }^{35}$ S]methionine for $30 \mathrm{~min}$ ) and/or the chase step (re-incubation with unlabelled methionine): ( $\$$ ), oleate present in pulse step, but not chase step; $(\triangle)$, oleate present in both pulse and chase steps; $(\diamond)$, oleate absent in pulse step, but present in the chase step; (A), oleate absent from both pulse and chase steps. Values are the means and standard deviations represented by vertical bars for four experiments performed in duplicate. dpm, Disintegrations/min.

of apo-B across the RER membrane is not the only step regulating secretion. The effects of dietary fish oil are not irreversible, since hepatocytes from fish-oil-fed hamsters secrete apo-B in particles of the density of VLDL when degradation is inhibited by $o$-phenanthroline. It may be that a high level of $n-3$ fatty acids in the hepatocytes or the hepatocyte membranes slows the assembly of the VLDL precursor particle, perhaps by modifying the activity of MTP or altering synthesis of specific lipids. However, if degradation is inhibited, this may allow time for proper assembly to take place so that the particle can advance through the secretory pathway.

\section{Acknowledgements}

We would like to thank the BBBSRC and the British Heart Foundation for research support.

\section{References}

Boren J, Rustaeus S \& Olofsson S-O (1994) Studies on the assembly of apolipoprotein B-100 and B-48 containing very low density lipoproteins in McA-RH7777 cells. Journal of Biological Chemistry 269, 25879-25888.

Boren J, Rustaeus S, Wettesten M, Andersson M, Wiklund A \& Olofsson S-O (1993) Influence of triacylglycerol biosynthetic rate on the assembly of apo-B100 containing lipoproteins in Hep-G2 cells. Arteriosclerosis and Thrombosis 13, 1743-1754.

Boren J, Wettesten M, Sjoberg A, Thorlin T, Bondjer G, Wilklund O, Carlsson P \& Olofsson S-O (1990) Studies on the assembly of apo-B 100 containing lipoproteins in HepG2 cells. Journal of Biological Chemistry 263, 4434-4442.

Cartwright IJ, Hebbachi A-M \& Higgins JA (1993) Transit and sorting of apolipoprotein B within the endoplasmic reticulum and Golgi compartments of isolated hepatocytes from normal and orotic acid fed rats. Journal of Biological Chemistry 268, 20937-20952

Cartwright IJ \& Higgins JA (1992) Quantification of apolipoprotein B-48 and B-100 in rat liver endoplasmic reticulum and Golgi. Biochemical Journal 285, 153-159.

Cartwright IJ \& Higgins JA (1995) Intracellular events in the assembly of very low density lipoprotein lipids with apolipoprotein B in isolated rabbit hepatocytes. Biochemical Journal 310, 897-907.

Cartwright IJ \& Higgins JA (1996) Intracellular degradation in the regulation of secretion of apolipoprotein B-100 by rabbit hepatocytes. Biochemical Journal 314, 377-384.

Cartwright IJ, Higgins JA, Wilkinson J, Bellavia S, Kendrick JS \& Graham JM (1997) Investigation of the role of lipids in the assembly of very low density lipoproteins in rabbit hepatocytes. Journal of Lipid Research 38, 531-545.

Davis RA, Thrift RN, Wu CC \& Howell KE (1990) Apolipoprotein $B$ is both integrated and translocated across the endoplasmic reticulum membrane. Joumal of Biological Chemistry 26, 10006-10011.

Dixon JL, Furukawa S \& Ginsberg HN (1991) Oleate stimulates secretion of apolipoprotein-B containing lipoproteins from Hep-G2 cells by inhibiting early intracellular degradation of apolipoprotein B. Journal of Biological Chemistry 266, 50805086.

Dixon JL \& Ginsberg HN (1993) Regulation of hepatic secretion of apolipoprotein B containing lipoproteins; information obtained from cultured liver cells. Journal of Lipid Research 34, 167-179.

Du EZ, Kurth J, Wang S-L, Humiston P \& Davis RA (1994) Proteolysis coupled secretion of the $\mathrm{N}$ terminus of apolipoprotein-B. Journal of Biological Chemistry 269, 4169-4176.

Gibbons GF (1994) A comparison of in vitro models to study hepatic lipid and lipoprotein metabolism. Current Opinion in Lipidology 5, 191-198. 
Gordon DA, Jamit H, Gregg RE, Olofsson S-O \& Boren J (1996) Inhibition of the microsomal triglyceride transfer step blocks the first step of apolipoprotein B lipoprotein assembly but not the addition of the core lipids in the second step. Journal of Biological Chemistry 271, 33047-33053.

Gordon DA, Wetterau JR \& Gregg RE (1995) Microsomal triglyceride transfer protein: a protein complex required for the assembly of lipoprotein particles. Trends in Cell Biology 5, 317-332.

Higgins JA \& Hutson J (1984) The roles of Golgi and endoplasmic reticulum in the synthesis and assembly of lipoprotein lipids in rat hepatocytes. Journal of Lipid Research 25, 1295-1305.

Jamil H, Dickson JE, Ching-Hsuen C, Lago MW, Rinhhaert JK, Biller SA, Gregg RE \& Wetterau JR (1995) Microsomal triglyceride transfer protein: specificity of lipid binding and transport. Journal of Biological Chemistry 270, 6549-6554.

Kosykh VA, Surguchov AP, Podres EA, Novikov DK \& Sudarickoc (1988) VLDL apoprotein secretion and apo-B mRNA levels in primary culture of cholesterol loaded rabbit hepatocytes. FEBS Letters 232, 103-106.

Lang CA \& Davis RA (1990) Fish oil fatty acids impair VLDL assembly and/or secretion by cultured rat hepatocytes. Journal of Lipid Research 31, 2079-2086.

Packard CJ, Munro A, Lorimer AR, Gotto AM \& Shepherd J (1984) Metabolism of apolipoprotein B in large triglyceride rich very low density lipoproteins of normal and hypertriglyceridaemic subjects. Joumal of Clinical Investigation 74, 21782192.

Pullinger CR, North JD, Teng B-B, Rifici VA, Ronhild de Brito AE \& Scott J (1989) Apolipoprotein B gene is constitutively expressed in HepG2 cells: regulation of secretion by oleic acid, albumin and insulin and measurement of the mRNA half life. Journal of Lipid Research 30, 1065-1077.

Sato R, Imanaka T, Takatsuki A \& Takano T (1990) Degradation of newly synthesized apolipoprotein B by rat hepatocytes. Journal of Biological Chemistry 265, 11880-11884.

Shepherd J \& Packard CJ (1987) Metabolic heterogeneity in very low density lipoproteins. American Heart Joumal 113, 503508.

Sniderman AD \& Cianflone K (1993) Substrate delivery as a determinant of hepatic apo-B secretion. Arteriosclerosis and Thombosis 13, 629-636.

Wang H, Chen X \& Fisher EA (1993) n-3 Fatty acids stimulate intracellular degradation of apolipoprotein $\mathrm{B}$ in rat hepatocytes. Journal of Clinical Investigation 91, 1380-1389.

White AL, Graham DL, LeGros J, Pease RJ \& Scott J (1992) Oleate mediated stimulation of apolipoprotein B secretion from rat hepatoma cells. Journal of Biological Chemistry 267, $15657-15664$.

Wilkinson J, Higgins JA, Groot PHE, Gherardi E \& Bowyer DE (1992) Determination of the intracellular distribution and pool sizes of apolipoprotein B in rabbit liver. Biochemical Journal 288, 413-419.

Wilkinson J, Higgins IA, Groot PHE, Gherardi E \& Bowyer DE (1993) Topography of apolipoprotein B in subcellular fractions of rabbit liver probed with a panel of monoclonal antibodies. Journal of Lipid Research 34, 815-825.

Wu X, Zhou M, Huang L-S, Wetterau J \& Ginsberg HN (1996) Demonstration of a physical interaction between microsomal triglyceride transfer protein and apolipoprotein B during the assembly of apo-B containing lipoproteins. Journal of Biological Chemistry 271, 10277-10281.

Yeung SJ, Chen SH \& Chan L (1996) Ubiquitin proteosome pathway mediates intracellular degradation of apolipoprotein B. Biochemistry 35, 13843-13848.

Zhou M, Wu X, Huang L-S \& Ginsberg HN (1995) Apolipoprotein $\mathrm{B} 100$, an inefficiently translocated secretory protein is bound to the cytosolic chaperone heat shock protein 70 . Journal of Biological Chemistry 270, 25220-25224. 